\title{
Types and Indications of Colostomy and Determinants of Outcomes of Patients After Surgery
}

\author{
Abebe Engida ${ }^{1}$, Tsehay Ayelign ${ }^{1}$, Bekele Mahteme ${ }^{1}$, Tilahun Aida ${ }^{1}$, Berhane \\ Abreham ${ }^{1}$
}

ABSTRACT

BACKGROUND: Colostomy is one of the commonest life saving procedures done worldwide with an intention of either decompression of an obstructed colon or diversion of stool. Indications may vary from region to region. Attending morbidity and mortality is significant. the objective of this study was to Determine the common indications and types of colostomy, and outcomes of patients operated at SPHMMC

METHODS: A retrospective medical records of patients, operation log book and nursing records review was done in a two-year period between January 2011 and December 2013 at the College Teaching Hospital, St Paul's Hospital Millennium Medical, Addis Ababa.

RESULTS: During the two years, 253 colostomies were done and $219(86.6 \%)$ cases used for analysis. Of these, 151(68.9\%) were males. Age ranged from 15 to 85 years with a mean of 50.8. Most of the surgeries, 196(89.5\%), were done for emergency conditions. The three most common indications were gangrenous sigmoid volvulus, 102(46.6\%), colorectal cancers, 46(21.0\%, and abdominal injuries, 28(12.8\%). The commonest type of colostomy done was Hartman's colostomy, 179(81.7.1\%), gangrenous sigmoid volvulus being the predominant indication, 102(57\%). Loop colostomy constituted 35(16\%) of all the colostomies. Penetrating abdominal injuries was the main indication 15(42.9\%). Overall, 157 complications were seen on 106(48.4\%) patients. The most common complications were surgical site infection, 51(23.3\%), hospital acquired pneumonia, (10.5\%), and wound dehiscence, 17(7.8\%). The mortality rate was $9.6 \%(21)$.

CONCLUSION: Gangrenous sigmoid volvulus is the leading indication for colostomy. Mortality and morbidity rates are high. Aggressive resuscitation, early prompt operation and post op close follow-up should be emphasized.

KEYWORDS: Colostomy, Indication, Complications

DOI: http://dx.doi.org/10.4314/ejhs.v26i2.5

\section{INTRODUCTION}

Colostomy is exteriorization of part of the colon to the anterior abdominal wall. It is one of the most common life saving emergency procedures done worldwide $(1,2)$. Colostomy may be done for emergency or elective surgical conditions for the management of wide ranges of congenital and acquired benign or malignant gastrointestinal conditions for two main purposes: diversion of the colon or decompression of the colon $(2,3)$. Diversion is done to protect contamination of the distal large bowel segment by stool and its attending complications. Diversion is done commonly for trauma or distal rectal elective surgeries (3). Decompression is done to relieve obstructed large bowel. Examples include sigmoid volvulus and malignant left side tumors $(4,5)$.

In the developing world, benign condition predominates, including sigmoid volvulus traumas and ileosigmoid knotting. Colorectal cancers also take a good share $(6,7)$. Colostomy has

${ }^{1}$ St Paul's Hospital Millennium Medical College, Addis Ababa, Ethiopia Corresponding Author: Abebe Engida, Email: engidaabebe@yahoo.com 
contributed significantly to reduction in abdominal trauma related deaths (5). In the developed world, malignant colorectal cancers with or without obstruction are common indications. Diverticulitis and ulcerative colitis are also among the common reasons $(2,4,8)$.

Depending on the way constructed colostomies are classified into four main types; Hartman's, loop, double barrel and spectacle. The choice of the type of colostomy depends on the indication, the experience of the surgeon and the patient's general condition during surgery (2). Hartman's end colostomy and loop colostomy are constructed frequently (6).

Colostomies can be temporary or permanent. Temporary colostomies will be reversed after sometime when the patient's condition and the reason for the colostomy is done allow (9). Permanent colostomies are indicated when abdominoperineal resection is done, the cancer is unresectable or the sphincter is damaged beyond repairable (10). Although it is a life saving procedure, both its construction and reversal have significant morbidity and mortality $(2,4,11)$. Complications can be related to the colostomy itself or the indication for it. Common early complications include surgical site infection, wound dehiscence, colostomy necrosis and retraction (6,12). Awareness of the common indications plus the types and the complications may help in improving outcomes of patients. The purpose of this study was to learn more about issues of our patient population for whom colostomies were done.

\section{METHODS AND MATERIAL}

A retrospective analysis of records of all patients who underwent colostomy from January 2011 to December 2013 at St. Paul's Hospital Millennium Medical College (SPHMMC) was conducted in April 2014. SPHMMC is a referral tertiary level teaching hospital in Addis Ababa, Ethiopia. The hospital accepts referrals and walking emergency conditions from all corners of the nation. Operation theater log book and individual patients' medical records were used as sources of data. Data were collected in a pretested data collection format. The data was checked for completeness, cleaned, coded, entered and analyzed with SPSS version 20. The associations of different variables related to complications of colostomy were tested for significance in chi square analysis. Considering a confidence level of $95 \%$, a P-value of $<0.05$ was considered significant in all statistical comparisons.

\section{RESULTS}

Socio-demographic Data: During the two years, 253 colostomies were done. Thirty-four cases were excluded for various reasons (17 incomplete recordings, 13 missing and 4 pediatrics). The remaining $219(86.6 \%)$ charts were used for analysis. Of these, 151(68.9\%) were males with a male to female ratio of 2.2:1. Age ranged from 15 to 85 years with a mean of 50.8. Most of the patients, 159(72.6\%), came from outside Addis Ababa (Table 1).

Indications and Type of Colostomy: In general, emergency surgical conditions make the majority of the reason for colostomy, 196(89.5\%). The common indications for colostomy in decreasing order were gangrenous sigmoid volvulus, 102(46.6\%), colorectal cancers, 46(21.0\%), abdominal injuries, 28(12.8\%, ileosigmoid knotting, 17(7.8\%) and advanced anorectal cancers, 6(2.7\%). Gangrenous colocolic intussusceptions, intra operative bowel injury, perineal injury and anastomotic leak each accounted for $5(2.3 \%)$ of the cases (Table 2). Among patients for whom colostomies were done following abdominal injury penetrating injuries were the main reason, 25 (89.3\%). Of these, bullet injury accounted for $15(60 \%)$, while stabbing constituted $10(40 \%)$.

The commonest type of colostomy done was Hartman's end colostomy, 179(81.7\%) (Figure 1), Gangrenous sigmoid volvulus being the predominant indication 102(57\%) followed by colorectal cancer, 37(20.7\%), ileosigmoid knotting, 17(9.5\%), and penetrating abdominal injuries $8(4.5 \%)$ (Figure 1; Table 3). Loop colostomy made up $35(16 \%)$ of all the colostomies, penetrating abdominal injuries were the main indication, $15(42.9 \%)$ followed by colorectal cancer, $7(20 \%)$. Perineal injury and iatrogenic (intra-operative) colon injury each made up 4(11.4\%) of the cases (Figure 1; Table $3)$. Double barrel and spectacle colostomies together accounted for only 4(2.3\%). 
Table 1: Socio-demographic characteristics of patients who had colostomy between January 2011 and

December 2013 at St Paul's Hospital Millennium Medical College, Addis Ababa, Ethiopia

\begin{tabular}{llll}
\hline Characteristics & Category & No & Percent \\
\hline \multirow{3}{*}{ Sex } & Male & 151 & 68.9 \\
& Female & 68 & 31.1 \\
\multirow{5}{*}{ Age in group } & 15-20 years & 7 & 3.2 \\
& 21-29 years & 22 & 10.0 \\
& 30- 39 years & 39 & 17.9 \\
Residence & 40-49 years & 37 & 17.1 \\
& 50-59 years & 36 & 16.2 \\
Occupation & 60 years and above & 78 & 35.6 \\
& Addis Ababa & 60 & 27.4 \\
& Outside AA & 159 & 72.6 \\
Socioeconomic status & Farmers & 184 & 84.0 \\
& Government employee & 15 & 7.0 \\
& Self employed & 20 & 9.0 \\
& Low & 175 & 80.0 \\
& Middle & 29 & 13.2 \\
\hline
\end{tabular}

Table 2. Indication for colostomy for patients whose colostomy was constructed between January 2011 and December 2013 at SPHMMC, Addis Ababa, Ethiopia.

\begin{tabular}{lll}
\hline Indication for colostomy & No & \% \\
\hline Gangrenous sigmoid Volvulus & 102 & 46.5 \\
Colorectal cancer & 46 & 21.0 \\
Penetrating abdominal injury & 25 & 11.4 \\
Ileosigmoid Knotting & 17 & 7.8 \\
Anorecatl cancer & 6 & 2.7 \\
Intussusceptions & 5 & 2.3 \\
Perineal injury & 5 & 2.3 \\
Intraop bowel injury & 5 & 2.3 \\
Anastomotic leak & 5 & 2.3 \\
Blunt abdominal injury & 3 & 1.4 \\
Total & 219 & 100 \\
\hline
\end{tabular}

Table 3: Types and indications of colostomy, between January 2011 and December 2013 at St Paul's Hospital Millennium Medical College, AA, Ethiopia.

\begin{tabular}{|c|c|c|c|}
\hline $\begin{array}{l}\text { Type of } \\
\text { colostomy }\end{array}$ & Indication & No & $\%$ \\
\hline \multirow[t]{9}{*}{ End } & $\begin{array}{l}\text { Gangrenous } \\
\text { volvulus }\end{array}$ & 102 & 57.0 \\
\hline & Colorectal cancer & 37 & 20.7 \\
\hline & Ileosigmoid Knotting & 17 & 9.5 \\
\hline & $\begin{array}{l}\text { Penetrating abdominal } \\
\text { injury }\end{array}$ & 8 & 4.5 \\
\hline & Intussusceptions & 5 & 2.8 \\
\hline & Anastomotic leak & 5 & 2.8 \\
\hline & Anorectal cancer & 4 & 2.2 \\
\hline & Perineal injury & 1 & 0.6 \\
\hline & Total & 179 & 100 \\
\hline \multirow[t]{8}{*}{$\begin{array}{l}\text { Loop } \\
\text { colostomy }\end{array}$} & $\begin{array}{l}\text { Penetrating abdominal } \\
\text { injury }\end{array}$ & 15 & 42.9 \\
\hline & Colorectal cancer & 7 & 20 \\
\hline & $\begin{array}{l}\text { Intera operative bowel } \\
\text { injury }\end{array}$ & 4 & 11.4 \\
\hline & Perineal injury & 4 & 11.4 \\
\hline & Blunt abdominal injury & 3 & 8.6 \\
\hline & anorectal cancer & 2 & 5.7 \\
\hline & Total & 35 & 100 \\
\hline & $\begin{array}{l}\text { Penetrating abdominal } \\
\text { injury }\end{array}$ & 3 & 75 \\
\hline Double & Colorectal cancer & 1 & 25 \\
\hline barrel & Total & 4 & 100 \\
\hline Spectacle & Colorectal cancer & 1 & 100 \\
\hline
\end{tabular}




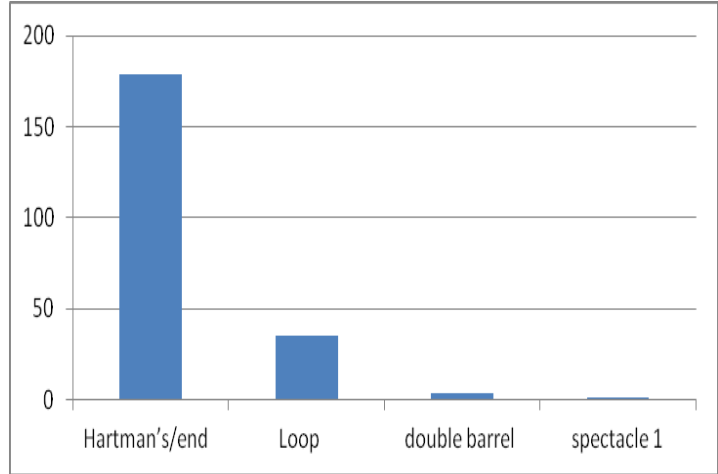

Figure 1: Types of colostomy and their frequency, between January 2011 and December 2013 at St Paul's Hospital Millennium Medical College, AA, Ethiopia.

Complications and Outcome: Complications were seen on $106(48.4 \%)$ patients: in $71(67.0 \%)$ males and 35(33.0\%) females. There was no statistically significant difference in the gender and age groups. Overall, 157 complications were seen. The most common complications were surgical site infection, 51(23.3\%), hospital acquired pneumonia, (10.5\%), wound dehiscence, 17(7.8\%), adhesion obstruction, 15(6.8\%) and colostomy retractions, $13(5.9 \%)$ (Table 4). Rate of complication was higher in those who had loop colostomy, $57.1 \%$ (20 of 35 patients), compared to patients who had Hartman's 46.4\% (83 of 179 patients).

Table 4: Type and rate of complications among patients who had colostomy, between January 2011 and December 2013 at St Paul's Hospital Millennium Medical College, AA, Ethiopia

\begin{tabular}{lll}
\hline Type of complication* & No & \% \\
\hline Surgical site infection including & 51 & 23.3 \\
paracolostomy infection & & \\
Hospital acquired pneumonia & 23 & 10.5 \\
Wound dehiscence & 17 & 7.8 \\
Adhesion obstruction & 15 & 6.8 \\
Colostomy retractions & 13 & 5.9 \\
Colostomy necrosis & 10 & 4.6 \\
Colostomy diarrhea & 6 & 2.7 \\
Colostomy Prolapse & 6 & 2.7 \\
GI onset sepsis & 5 & 2.3 \\
Paracolostomy hernia & 3 & 1.4 \\
ARF- prerenal azothemia & 3 & 1.4 \\
Dvt & 3 & 1.4 \\
Stomal stenosis & 2 & 0.9 \\
& 157 & 71.7 \\
\hline
\end{tabular}

*more than one complication seen on one patient Among the patients with gangrenous sigmoid volvulus, 48(47.1\%) developed complications. The figures for colorectal cancer, penetrating abdominal injury, ileosigmoid knotting and anorectal cancer were 29(63.0\%), 11(44\%), $4(66.7 \%), 5(29.4 \%)$ and $4(66.6 \%)$ respectively. The indication for colostomy was significantly associated with the occurrence of complication $(\mathrm{P}=0.024)$.

Totally, 198 of the patients were discharged improved, while 21 died making a mortality rate of $9.6 \%$. Multi-organ dysfunction syndrome was the most common cause of death, 13(62.0\%). Among patients who died of MODS, 6(46.2\%) presented with gangrenous sigmoid volvulus, while $3(23.1 \%)$ presented with colorectal cancer (Table 5). Two of the three patients who died of Myocardial infarction presented with gangrenous sigmoid volvulus and one with foreigner's gangrene extending to the bowel. All the three were diabetic.

There was a significant association $(\mathrm{P}=0.000)$ between associated chronic illness and mortality. There were 36 hypertensive patients of whom two died. Out of eight diabetic patients four of them died. Three of five patients with both hypertension and diabetes died. There was one patient with leukemia who also died.

Table 5: Causes of death and the indication for colostomy among patients who underwent colostomy operation, between January 2011 and December 2013 at St Paul's Hospital Millennium Medical College, AA, Ethiopia.

\begin{tabular}{l|lll}
\hline Cause & Indication & No & \% \\
\hline MODS & Gangrenous sigmoid & 6 & 46.2 \\
& Colorectal cancer & 3 & 23.1 \\
& Anorectal cancer & 1 & 7.7 \\
& Ileosigmoid knotting & 1 & 7.7 \\
& Penetrating abdominal & 1 & 7.7 \\
injury & & \\
& Intussusceptions & 1 & 7.7 \\
Myocardial & Total & 13 & $62.0 \%$ \\
infarctions & Gangrenous sigmoid & 2 & 66.7 \\
Respiratory & Fournier's gangrene & 1 & 33.3 \\
failure & Total & 3 & 14.3 \\
& Colorectal cancer & 2 & 66.7 \\
Unknown & Anorectal cancer & 1 & 33.3 \\
& Total & 3 & 14.3 \\
& Gangrenous sigmoid & 1 & 50 \\
& Colorectal cancer & 1 & 50 \\
& Total & 3 & 14.3 \\
\hline
\end{tabular}




\section{DISCUSSION}

Socio-demographic Factors: The hospital in which this study was conducted receives patients from all parts of the country and fairly represents the nation's picture. Similar to other literatures, males and old age groups are more commonly affected for the conditions necessitating colostomy (6). This is because the conditions are the disease of the elderly although no age group is exempted. In this study, the mean age of the study population was 50.79. This is comparable with the report of studies done in India and Pakistan where the mean ages were 50.5 and 55 years. $(13,14)$. There was male predominance with a male to female ratio of 2.2:1, comparable to another study in Tikur Anbessa (6). This figure is lower than those seen in India (7:3) and Pakistan (6.5:1) $(13,14)$.

Indication and Type of Colostomy: This study revealed that the majority of the colostomies were done for emergency conditions, similar to findings in other literatures $(6,12)$. The three most common reasons for colostomy construction were gangrenous sigmoid volvulus, left side colorectal cancers and trauma in that order. Similar findings were also reported elsewhere (6). It was found that Gangrenous sigmoid volvulus was the most common indication necessitating colostomy. This is not a surprise as Ethiopia is among countries with a high burden of sigmoid volvulus and late presentation is common $(15,16)$. This is similar with the report of a retrospective analysis done at a different hospital in Addis Ababa (6). Even in countries with high rate of sigmoid volvulus, nonsigmoid causes are reported to be common indications. For example, in Pakistan penetrating abdominal injuries were the most common causes (14).

Because the common indications were gangrenous sigmoid volvulus, (Hartman's colostomy is the procedure of choice) and left side colorectal tumors, Hartman's (end) colostomy was the most common type of colostomy (17). Hartman's colostomy is the most common form done worldwide. Reports in developing and developed nations show similar findings $(6,13)$. Loop colostomy is most often done for trauma cases like the case in other sites. It was done more often for trauma and inoperable tumors. Protective Loop colostomies for distal rectal surgeries are very common indications elsewhere, but were not seen in our case because low or extended low resection was not frequent. Other forms of colostomies are not often in this study and other literatures too (6).

Complication and Outcome: Colostomy construction has complications due to the indication for which it is done and/or the construction itself $(4,6)$. Most of the colostomies were done for acute/emergency surgical conditions. Emergency surgeries are known for their high rate of morbidity and mortality $(18,19)$. That is the case in our study, a complication rate of $48.4 \%$. Similar high rates of complication were reported in Boston, -(47.6\%), Zurich, $-76 \%$ and Lahore, $-41.77 \%,(2,5,11,13)$.

Loop colostomy has the highest rate of complication compared to the other types of colostomy probably because it is done mostly for traumas, both penetrating and blunt injuries and technical difficulty. In trauma, contamination happens in two ways: the instrument causing the injury and the resulting fecal contamination/peritonitis. A similar high rate of complications in loop colostomies was reported by Wahl. (3).

Complications were infections or results of infection. Most common complications were surgical site infections (SSI) and pneumonia. SSI can be explained by bacterial translocation in gangrenous bowel and fecal contamination in traumatic or spontaneous malignant perforations, anastomotic leak and intra-operative accidents. Similar findings were also reported elsewhere. Old age and some degree of immunosuppression can also contribute to cancers.

The outcome of construction of colostomy had a significant association with the presence of associated chronic illness $(\mathrm{P}=0.000)$. This finding is similar with a report from USA that showed a statistically significant increase in morbidity with the presence of diabetes $(\mathrm{P}=0.036)(20)$. The mortality rate was high $(9.5 \%)$, largely due to late presentation and associated co-morbidities. As most patients came from out of Addis Ababa, late presentation is likely. The rate of mortality, though high, is not far from reports in the literature which ranges from 3-20\% $(2,4,21)$.

In conclusion, Gangrenous sigmoid volvulus, colorectal cancer and trauma are the leading indications for colostomy. Attending mortality and morbidity are significant and are mostly infection 
related. Aggressive resuscitation, early prompt operation and post operative close follow-up should be given great emphasis. Optimal and aggressive management of co-morbidity might also help in reducing mortality.

\section{REFERENCES}

1. F. Charies Brunicardi, Dana K. Andersen, Timothy R. Billiar, David L. Dunn, John G. Hunter, Raphael E. Pollock: Schwartz's principle of surgery, $9^{\text {th }}$ edition: The McGrawHill Companies, 2011

2. Marin AW, Di Giorgio A, Destito C, Mercuri M and etals. Typology of defunctioning colostomy and state of art in the treatment of bowel emergencies. Ann Ital Chir. 2005 MarApr;76(2):157-60.

3. Wahl $\mathrm{W}^{1}$, Hassdenteufel A, Hofer B, Junginger T. Temporary colostomies after sigmoid colon and rectum interventions--are they still justified?. Langenbecks Arch Chir. 1997;382(3):149-56.

4. Miles RM, Greene RS. Review of colostomy in a community hospital. Am J Surg. 1983;49(4):182-6.

5. Bugis SP, Blair NP, Letwin ER. Management of blunt and penetrating colon injuries. Am J Surg 1992; 163:547-50.

6. Bekele $A^{1}$, Kotisso B, Tesfaye M. Patterns and indication of colostomies in Addis Ababa, Ethiopia. Ethiop Med J. 2009 Oct; 47(4):285.

7. Boland, Elena; Hsu, Allen; Brand, Marc I.; Saclaride, Theodore J. Indications and complications of colostomy in Lagos, Nigeria. 2007.

8. Robert Krouse, Marcia Grant, Betty Ferrell and etals. Quality of Life Outcomes in 599 Cancer and Non-Cancer Patients with Colostomies. Journal of Surgical Research 2007;138, 79-87

9. Garber HI, Morris DM, Eisenstat TE, Coker DD, Annous MO. Factors influencing the morbidity of colostomy closure. Dis Colon Rectum. 1982; 25(5):464-70.

10. W. Brian Perry, \& J.Christopher Connaughton. Abdominoperineal Resection: How is it Done and What are the Results? Clin Colon Rectal Surg. Aug 2007; 20(3): 213-220.

11. Mirelman D, Corman ML, Veidenheimer MC, Coller JA. Colostomies--indications and contraindications: Lahey Clinic experience, 1963--1974. Dis Colon Rectum. 1978; 21(3):172-6.
12. Safir Ullah, Naeem Mumtaz, Mian Asad Ullah, Siddique Ahmad. COMPLICATIONS OF INTESTINAL STOMAS. Journal of postgraduate medical institute 2005.19(4)

13. Zeeshanuddin Ahmad, Apoorv Sharma, Pradeep Saxena, Anuradha Choudhary, Minhajuddin Ahmed. A clinical study of intestinal stomas: its indications and complications. Int J Res Med Sci. 2013;1(4):536-540.

14. Qamar A. Ahmad, M. Kamran Saeed, Mah Jabeen Muneera M. Sarfraz Ahmed and Kamran Khalid. Indications and complications of intestinal stomas - A tertiary care hospital experience in Lahore. Pakistan. Biomedica. 2010; 26:144-47.

15. Ali MK. Treatment of sigmoid volvulus: experience in Gondar, north-west Ethiopia. Ethiop Med J. 1998 Jan; 36 (1):47-52.

16. Shepherd, J. J. The epidemiology and clinical presentation of sigmoid volvulus. Br J Surg, 1969; 56:353-359.

17. Jonathan C Samuel,Adesola Akinkuotu, Nelson Msiska, Bruce A Cairns, Arturo P Muyco, and Anthony G Charles. Re-examining treatment strategies for sigmoid volvulus: An analysis of treatment and outcomes in Lilongwe, Malawi. Glob j Surg. 2010 Oct; 1(2):149-153.

18. Lane Smothers ,Linda Hynan, Jason Fleming, Richard Turnage, Clifford Simmang ,Thomas Anthony. Emergency Surgery for Colon Carcinoma. Dis Colon Rectum 2003;46(1):2430.

19. Arnaud Alves, Yves Panis, Pierre Mathieu, \& etals. Postoperative Mortality and Morbidity in French Patients Undergoing Colorectal Surgery: Results of a Prospective Multicenter Study. Arch Surg. 2005; 140 (3):278-283.

20. Ghorra SG, et al. closure: impact of preoperative risk factors on morbidity. Am J Surg. 1999 Mar; 65(3):266-9.

21. Durkaya Ören, S. Selçuk Atamanalp, Bülent Aydinli \& etals. An Algorithm for the Management of Sigmoid Colon Volvulus and the Safety of Primary Resection: Experience; with 827 Cases. Dis Colon Rectum. 2007; 50(4):489-497. 